\title{
Evening exercise is associated with lower odds of visual field progression in Chinese patients with primary open angle glaucoma
}

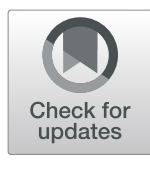

Xiafei Pan ${ }^{1,2 \dagger}$, Kai Xu ${ }^{3 \dagger}$, Xin Wang ${ }^{2,4}$, Guofu Chen ${ }^{2,4}$, Huanhuan Cheng ${ }^{2,4}$, Alice Jia Liu ${ }^{2,4}$, Laurence Tang Hou ${ }^{5}$, Lin Zhong ${ }^{2,4}$, Jie Chen ${ }^{2 *}$ (i) and Yuanbo Liang ${ }^{2,4^{*}}$

\begin{abstract}
Background: Exercise is widely known to lower intraocular pressure and increase ocular blood flow, which may be beneficial for glaucoma management. However, there are few studies that have reported on the relationship between exercise and glaucoma progression. The aim of our study was to investigate the exercise habits of those with primary open angle glaucoma (POAG) and its association with the progression of visual field (VF) loss.

Methods: Daily physical activity (PA) was monitored by an accelerometer (ActiGraph wGT3x-BT) which patients wore for more than $10 \mathrm{~h}$ of being awake on their right wrists for 1 week.

Results: Seventy-one non-progressive and 27 progressive patients were enrolled in the study. 24-h moderate to vigorous physical activity (MVPA) exercise showed that POAG patients had similar variation trends consisting of 3 wave peaks and 2 wave hollows. Minutes spent in MVPA was $19.89 \pm 15.81$ and 21.62 \pm 15.10 during 07:00-09:00 h $(p=0.204), 15.40 \pm 14.49$ and $15.67 \pm 12.43$ during 15:00-17:00 $\mathrm{h}(p=0.822)$ and $17.26 \pm 21.11$ and $11.42 \pm 11.58$ during 18:00-20:00 $\mathrm{h}(p=0.001)$ in the non-progressive and progressive group, respectively. Univariate analysis indicated that $10 \mathrm{~min}$ of MVPA (18:00-20:00 h) [odds ratio, OR $(95 \% \mathrm{Cl})=0.82(0.73,0.92)$ ], average mean arterial pressure [OR $(95 \% \mathrm{Cl})=0.96(0.94,0.98)]$, age $[\mathrm{OR}(95 \% \mathrm{Cl})=1.06(1.03,1.08)]$, male $[\mathrm{OR}(95 \% \mathrm{Cl})=0.67(0.48,0.96)]$, spherical equivalent $[\mathrm{OR}(95 \% \mathrm{Cl})=1.14(1.07,1.22)]$ and IOP-lowering medications [OR $(95 \% \mathrm{Cl})=1.54(1.16,2.05)]$ were significantly correlated with having progressive VF damage. Multivariable analysis showed that 10 min of MVPA $(18: 00-20: 00 \mathrm{~h})[\mathrm{OR}(95 \% \mathrm{Cl})=0.85(0.75,0.97)]$ was associated with progressive VF loss even after adjusting for other risk factors.
\end{abstract}

Conclusions: Evening exercise may lower the odds of VF progression, suggesting that exercise habits possibly play an important role in glaucoma progression.

Keywords: Glaucoma, Exercise habits, Visual field progression

\section{Background}

Glaucoma is a multifactorial optic neuropathy with an unclear pathogenesis which can result in irreversible visual field (VF) damage [1]. The number of glaucoma patients ( 40 to 80 years old) is estimated to be 76.0 million by 2020 worldwide, and projected to increase to 111.8 million by 2040 [2]. Currently, intraocular pressure

\footnotetext{
*Correspondence: 675149282@qq.com; yuanboliang@126.com

${ }^{+}$Xiafei Pan and Kai Xu are co-first authors.

${ }^{2}$ School of Ophthalmology and Optometry, Eye Hospital, Wenzhou Medical University, No. 270, Xue Yuan Xi Road, Wenzhou 3250027, Zhejiang, China Full list of author information is available at the end of the article
}

(IOP) is the only modifiable risk factor for preventing glaucoma damage. However, patients diagnosed with normal tension glaucoma (NTG) and high-tension glaucoma (HTG) can still experience progressive VF loss even with a normal IOP. The Early Manifest Glaucoma Trial (EMGT) showed that 56\% of untreated NTG patients progressed during the 6-year follow up [3]. In the Collaborative Normal-Tension Glaucoma Study (CNTGS), about one third of untreated subjects had localized progression within a three-year span, and up to $50 \%$ within 5 to 7 years [4]. Forty-five percent of open angle glaucoma patients demonstrated VF progression in

(c) The Author(s). 2020 Open Access This article is distributed under the terms of the Creative Commons Attribution 4.0 International License (http://creativecommons.org/licenses/by/4.0/), which permits unrestricted use, distribution, and 
the EMGT study [5]. In addition, large IOP reductions do not necessarily stop the progression of NTG. A comparison of the spontaneous untreated group and lowered-IOP group (decreased by $30 \%$ from baseline) in the CNTGS study found that $35 \%$ of control eyes and $12 \%$ of treated eyes showed optic disk progression or VF loss [6]. It is clear that non-IOP factors played an important role in the development of the disease since glaucomatous progression was only slowed, rather than halted in subjects with normal IOP levels.

Physical activity (PA) is an essential part of daily life, with PA guidelines in the US advising adults to set aside time for at least $150 \mathrm{~min}$ to $300 \mathrm{~min}$ of moderate intensity PA per week or $75 \mathrm{~min}$ to $150 \mathrm{~min}$ of vigorous intensity aerobic PA per week to stay healthy [7]. The health benefits of PA have been widely documented for both systemic and ocular diseases including boosting the immune system, improving sleep quality, reducing the incidence of cardiac-cerebral vascular events and is correlated with decreased anxiety and depression rates [8-12]. For eyes in particular, exercise can decrease the IOP [13] and decreasing IOP is associated with the magnitude of exercise [14]. Moreover, choroidal blood flow increases substantially in primary open angle glaucoma (POAG) patients with elevated exercise-induced blood pressure [15]. However, there is little conclusive evidence proving the effectiveness of exercise in mitigating glaucomatous VF loss, with many studies collecting patients' physical activities through self-reported questionnaires $[16,17]$.

The objective of this study is to quantitatively monitor the daily PA of POAG patients and investigate the relationship between their exercise habits and progressive glaucomatous VF defect.

\section{Methods}

The study was approved by the Institutional Review Board of Wenzhou Medical University (KYK [2018]20). Participants gave written informed consent and completed the study procedures between August 2017 and June 2018. The study followed the tenets of the Declaration of Helsinki.

\section{Study participants}

Subjects were recruited from the Wenzhou Glaucoma Progression Study (WGPS), a longitudinal study exploring POAG patients' glaucomatous clinical features and progression. POAG was defined as (1) open angles on gonioscopy, (2) glaucomatous optic disc changes, including neuroretinal rim narrowing, notching and retinal nerve fiber layer defects, (3) repeatable VF defects, (4) above changes in the absence of any other identifiable cause. Subjects were excluded if they were less than 18 years of age or they had any history of intraocular surgery (except uncomplicated cataract or glaucoma surgery).

All participants underwent a comprehensive ophthalmologic examination in the Clinical and Epidemiological Eye Research Center of the Eye Hospital of Wenzhou Medical University involving a questionnaire on the use of IOP-lowering medications and past medical history, gonioscopy, automated refraction (WAM-5500, Grand Seiko, Japan), Goldmann applanation tonometry (HAAGSTREIT 900 CM, Swiss), VF testing (Humphrey Field Analyzer IIi (HFA IIi, Carl Zeiss Meditec Inc., Dublin, CA), optical coherence tomography (OCT, Carl Zeiss Cirrus HD-OCT 4000, Germany), Lenstar (HAAG-STREIT LS900, Swiss), blood pressure (BP) measurements (Omron Automatic BP instrument (model HEM-7136, Omron Healthcare, Inc., IL) and other basic parameters. Patients were advised to follow up at the center every three to 6 months. Mean arterial pressure (MAP) was calculated as $(1 / 3$ systolic $\mathrm{BP}+2 / 3$ diastolic $\mathrm{BP})$ while average MAP (aMAP) was the average MAP during all visits (from the baseline to the most recent visit). Mean IOP (mIOP) was obtained by averaging the IOP from all visits. PA was also measured during the follow-ups.

A total of 106 participants took part in the crosssectional study. There were seven $(6.60 \%)$ subjects with poor compliance and one $(0.94 \%)$ subject whose device had technical issues. In the end, data from 98 patients were analyzed, including 27 patients in the progressive group and 71 patients in the non-progressive group. A valid day was defined as more than $10 \mathrm{~h}$ of awake wear time [18], and patients with less than six valid days were excluded from the study.

\section{Evaluation of physical activity}

The accelerometer, ActiGraph wGT3x-BT (LLC, Pensacola, FL, USA), is an important tool that can accurately measure a person's daily PA intensity and duration [19, 20]. Participants' daily PA was recorded by the accelerometer (worn on the right wrist) for 1 week [21, 22]. Subjects were instructed on proper usage of the device and informed to take the device off when swimming or showering. Patients were also advised to wear the accelerometer for the entire day unless it was uncomfortable, or that it affected their sleep. Main measures of PA included calories burned per day, light PA (LPA) time per day, moderate PA (MPA) time per day, vigorous $\mathrm{PA}$ (VPA) time per day, very vigorous PA (VVPA) time per day, moderate to vigorous physical activity (MVPA) time per day and step counts. ActiLife software (version 6.13.3; ActiGraph, Pensacola, FL) was used to process the raw data and the values were presented as total daily and hourly counts per minute. Acceleration for $\mathrm{x}-, \mathrm{y}-$, and $\mathrm{z}$-axes and vector magnitude (VM) were converted to $10 \mathrm{~s}$ epochs. Kcals was calculated by Freedson VM3 
Combination (2011), $\mathrm{kcals}=\mathrm{S} \times[0.00097 \times \mathrm{VM} \quad(\mathrm{x}, \mathrm{y}$, $\mathrm{z})+(0.08793 \times \mathrm{BM})]-5.01582, \mathrm{~S}=$ Duration of exercise $(\mathrm{s}) /$ $60 \mathrm{~s}, \mathrm{BM}=$ Body Mass $(\mathrm{kg})$ [23]. We used the validated Sasaki's cut-point sets to estimate the amount of PA time: LPA, $\leq 2690$ bouts; MPA, 2691 6166 bouts; VPA, 6167 9642 bouts; VVPA, $\geq 9643$ bouts [24]. During this period, subjects were reminded to maintain their daily lifestyle and to not deliberately increase their total amount of exercise. Subjects were queried by phone call to verify the estimated wear time if any doubts about their compliance arose.

\section{Detection of visual field}

Participants recruited from the WGPS needed to have at least 4 regular, reliable VF examinations $(<20 \%$ fixation losses and $<15 \%$ false-positive results). Humphrey VF testing was performed using the 24-2 Swedish Interactive Thresholding Algorithm (Standard) with stimulus size III after near refractive correction.

\section{Definition: progressive VF defect}

Progressive VF loss was defined as statistically significant deterioration $(p<0.05)$ on the pattern deviation change probability maps in the same three or more points with confirmation by at least two consecutive visits using the event-based glaucoma change probability (GCP) analysis of the Humphrey field analyzer in the Forum Glaucoma Workplace (Zeiss Carl-Forum 4.0) [25, 26].

Recruited glaucoma subjects were divided into the progressive and non-progressive group depending on the nature of their disease. Daily PA was compared between these two groups. Patients were classified as a progressor if they had at least one eye with progressive VF loss. Data for both eyes were obtained, and for the nonprogressive group, the worse eye was chosen. For patients with two progressive eyes, the worse progressive eye was chosen, and for patients with only one progressive eye, the progressive eye was selected. The average data of all visits, such as IOP and MAP were used in the study. The presenting visual acuity at the time of PA assessment was used and data involving other parameters were from the baseline.

\section{Statistical methods}

Statistics were performed using SPSS (version 21.0). A $p$ value of less than 0.05 was considered statistically significant. Demographics, ocular characteristics, and PA are presented using mean and standard deviation and compared between the non-progressive and progressive group using an independent sample t-test for normallydistributed continuous variables, the Mann-Whitney U test for non-normally distributed continuous variables, and a Chi-squared test for categorical variables. Logistic regression was used to explore the association of binary dependent variable "progression of glaucoma" with continuous or categorical independent variables.

\section{Results}

Seventy-one patients with non-progressive VF loss $(61.45 \pm 12.99$ years, $53.52 \%$ male $)$ and 27 patients with progressive VF loss (67.22 \pm 8.93 years, $44.44 \%$ male) who had no less than 6 days of valid accelerometer data were enrolled from the WGPS. POAG patients with and without progressive VF damage were similar in gender, body-mass index, mIOP, aMAP, visual field MD, retinal nerve fiber layer, presenting visual acuity, spherical equivalent, axial lengths, central corneal thickness, IOPlowering medications, self-reported hypertension, selfreported diabetes, total days of PA measurement, followup period and number of follow-ups $(p>0.05$ for all), but differed in age $(p=0.023)$ (Table 1$)$.

Median calories burned per day was $3563.44 \pm 1832.82$ $\mathrm{kcal}$ and $2909.29 \pm 1250.14 \mathrm{kcal}$ in the non-progressive and progressive group, respectively $(p=0.111)$. A comparison of POAG patients' total PA duration for all exercise levels (light, moderate, vigorous, very vigorous and MVPA) revealed no significant differences between the two groups $(p>0.05$ for all). There was also no significant difference for total step count between the two groups $(p=0.617)$ (Table 2$)$.

The line graph illustrates the variation of MVPA time subjects spent over a whole day. MVPA fluctuations for the non-progressive and progressive groups had similar trends in variation ( 3 wave peaks and 2 wave hollows). The average number of minutes patients spent in MVPA during 07:00-09:00 $\mathrm{h}$ was $19.89 \pm 15.81$ and 21.62 \pm $15.10(p=0.204), 15.40 \pm 14.49$ and $15.67 \pm 12.43$ during $15: 00-17: 00 \mathrm{~h}(p=0.822)$, and $17.26 \pm 21.11$ and $11.42 \pm$ 11.58 during $18: 00-20: 00 \mathrm{~h}(p=0.001)$ in the nonprogressive and progressive group, respectively (Fig. 1).

To investigate other possible factors influencing progressive VF loss of POAG patients, we conducted a binary logistic regression (univariate and multivariable analysis). Univariate analysis indicated that $10 \mathrm{~min}$ of MVPA (18:00-20:00 h) [Odds ratio, OR $(95 \% \mathrm{CI})=0.82$ $(0.73,0.92)]$, average mean arterial pressure (aMAP) [OR $(95 \% \mathrm{CI})=0.96(0.94,0.98)]$, age $[\mathrm{OR}(95 \% \mathrm{CI})=1.06$ $(1.03,1.08)]$, male $[\mathrm{OR}(95 \% \mathrm{CI})=0.67(0.48,0.96)]$, spherical equivalent (SE) $[\mathrm{OR} \quad(95 \% \mathrm{CI})=1.14 \quad(1.07$, 1.22)] and IOP-lowering medications [OR $(95 \% \mathrm{CI})=$ $1.54(1.16,2.05)]$ were significantly correlated with having progressive VF damage. Multivariable analysis showed that the following features were associated with progressive VF defect: 10 min spent in MVPA [OR (95\% $\mathrm{CI})=0.85(0.75,0.97)]$, aMAP $[\mathrm{OR}(95 \% \mathrm{CI})=0.95(0.93$, 0.97)], age $[\mathrm{OR}(95 \% \mathrm{CI})=1.08(1.05,1.11)]$, SE [OR $(95 \% \mathrm{CI})=1.11(1.02,1.20)]$ and lowering-IOP medications $[\mathrm{OR}(95 \% \mathrm{CI})=2.95(2.02,4.31)]$ (Table 3$)$. 
Table 1 Demographics and ocular characteristics of non-progressive and progressive patients

\begin{tabular}{|c|c|c|c|}
\hline Parameter & $\begin{array}{l}\text { Non-progressive } \\
\text { group }(n=71)\end{array}$ & $\begin{array}{l}\text { Progressive group } \\
(n=27)\end{array}$ & $p$ value \\
\hline Gender (\% male) & $38(53.52 \%)$ & $12(44.44 \%)$ & $0.422^{\mathrm{a}}$ \\
\hline Age (yrs) & $61.45 \pm 12.99$ & $67.22 \pm 8.93$ & $0.023^{b}$ \\
\hline BMI $\left(\mathrm{kg} / \mathrm{m}^{2}\right)$ & $24.32 \pm 3.09$ & $23.27 \pm 3.39$ & $0.143^{c}$ \\
\hline $\mathrm{mlOP}(\mathrm{mmHg})$ & $15.30 \pm 2.68$ & $15.39 \pm 3.71$ & $0.900^{c}$ \\
\hline aMAP (mmHg) & $94.00 \pm 8.43$ & $90.81 \pm 9.28$ & $0.107^{c}$ \\
\hline Visual field MD (dB) & $-7.44 \pm 5.24$ & $-6.71 \pm 5.98$ & $0.233^{b}$ \\
\hline RNFL $(\mu \mathrm{m})$ & $76.54 \pm 12.93$ & $76.56 \pm 14.16$ & $0.995^{c}$ \\
\hline Presenting visual acuity (logMAR) & $0.23 \pm 0.20$ & $0.28 \pm 0.20$ & $0.222^{b}$ \\
\hline SE (D) & $-0.89 \pm 3.14$ & $0.06 \pm 2.74$ & $0.117^{\mathrm{b}}$ \\
\hline $\mathrm{AL}(\mathrm{mm})$ & $24.19 \pm 1.72$ & $23.86 \pm 1.05$ & $0.520^{\mathrm{b}}$ \\
\hline $\mathrm{CCT}(\mu \mathrm{m})$ & $539.07 \pm 30.18$ & $540.89 \pm 28.84$ & $0.788^{c}$ \\
\hline No. of visual field (MD) & & & $0.744^{\mathrm{a}}$ \\
\hline$M D \geq-12 d B[n(\%)]$ & $67(94.40 \%)$ & $25(92.60 \%)$ & \\
\hline$M D<-12 \mathrm{~dB}[\mathrm{n}(\%)]$ & $4(5.60 \%)$ & $2(7.40 \%)$ & \\
\hline IOP-lowering medications & & & $0.228^{a}$ \\
\hline Non-medications [n (\%)] & $52(73.20 \%)$ & $17(63.00 \%)$ & \\
\hline One medication [n (\%)] & $17(23.90 \%)$ & 9 (33.30\%) & \\
\hline Two medications [n (\%)] & $2(2.80 \%)$ & $0(0.00 \%)$ & \\
\hline Three medications [n (\%)] & $0(0.00 \%)$ & $1(3.70 \%)$ & \\
\hline Self-reported hypertension [n (\%)] & $21(29.6 \%)$ & $6(22.2 \%)$ & $0.467^{\mathrm{a}}$ \\
\hline Self-reported diabetes [n (\%)] & $30(42.3 \%)$ & $8(29.6 \%)$ & $0.252^{\mathrm{a}}$ \\
\hline Total days of PA measurement & $6.82 \pm 0.39$ & $6.70 \pm 0.47$ & $0.225^{b}$ \\
\hline Follow-up period (months) & $41 \pm 10.89$ & $31.11 \pm 10.32$ & $<0.001^{b}$ \\
\hline Number of Follow-ups (n) & $11.20 \pm 3.97$ & $6.85 \pm 2.58$ & $<0.001^{\mathrm{b}}$ \\
\hline
\end{tabular}

$B M I=$ body-mass index; $m I O P=$ mean intraocular pressure during all visits; $M D=$ mean deviation in the baseline; $R N F L=$ retinal nerve fiber layer detected by $O C T$; $\triangle M A P=$ average mean arterial pressure during all visits; $\log M A R=\log$ arithm of the minimum angle of resolution presenting visual acuity measured at the time of $\mathrm{PA}$ assessment; $S E=$ spherical equivalent; $A L=$ axial lengths; $C C T=$ central corneal thickness; No. of visual field $(M D)$, visual field MD was measured at the time of PA assessment, $\mathrm{MD} \geq-12 \mathrm{~dB}$, mild to moderate VF damage; $\mathrm{MD}<-12 \mathrm{~dB}$, serious VF damage; $P A=$ physical activity. Data are represented as mean $\pm \mathrm{SD}$

${ }^{\mathrm{a}}$ Chi-squared test, ${ }^{\mathrm{b}}$ Mann-Whitney U test, Independent Sample T test. Bold values indicate statistical significance with alpha set at 0.05

Table 2 Difference in physical activity time between non-progressive and progressive patients

\begin{tabular}{llll}
\hline Parameter & Non-progressive group $(n=71)$ & Progressive group $(n=27)$ & $p$ value \\
\hline Kcals (per day) & $3563.44 \pm 1832.82$ & $2909.29 \pm 1250.14$ & $0.111^{\mathrm{a}}$ \\
Light PA (min/day) & $1360.27 \pm 54.31$ & $1365.03 \pm 32.62$ & $0.611^{\mathrm{a}}$ \\
Moderate PA (min/day) & $80.82 \pm 41.20$ & $75.28 \pm 30.33$ & $0.527^{\mathrm{b}}$ \\
Vigorous PA (min/day) & $4.06 \pm 8.87$ & $1.43 \pm 1.32$ & $0.469^{\mathrm{a}}$ \\
Very vigorous PA (min/day) & $0.28 \pm 0.47$ & $0.20 \pm 0.26$ & $0.975^{\mathrm{a}}$ \\
MVPA (min/day) & $85.16 \pm 45.67$ & $76.91 \pm 31.24$ & $0.311^{\mathrm{b}}$ \\
Steps (per day) & $9738.42 \pm 4248.72$ & $9379.48 \pm 2632.88$ & $0.617^{\mathrm{b}}$ \\
\hline
\end{tabular}

Kcal = calories burned after exercise; $P A=$ physical activity; MVPA = moderate to vigorous physical activity. Data are represented as mean \pm SD. ${ }^{a}$ Mann-Whitney U test. ${ }^{b}$ Independent Sample T test 


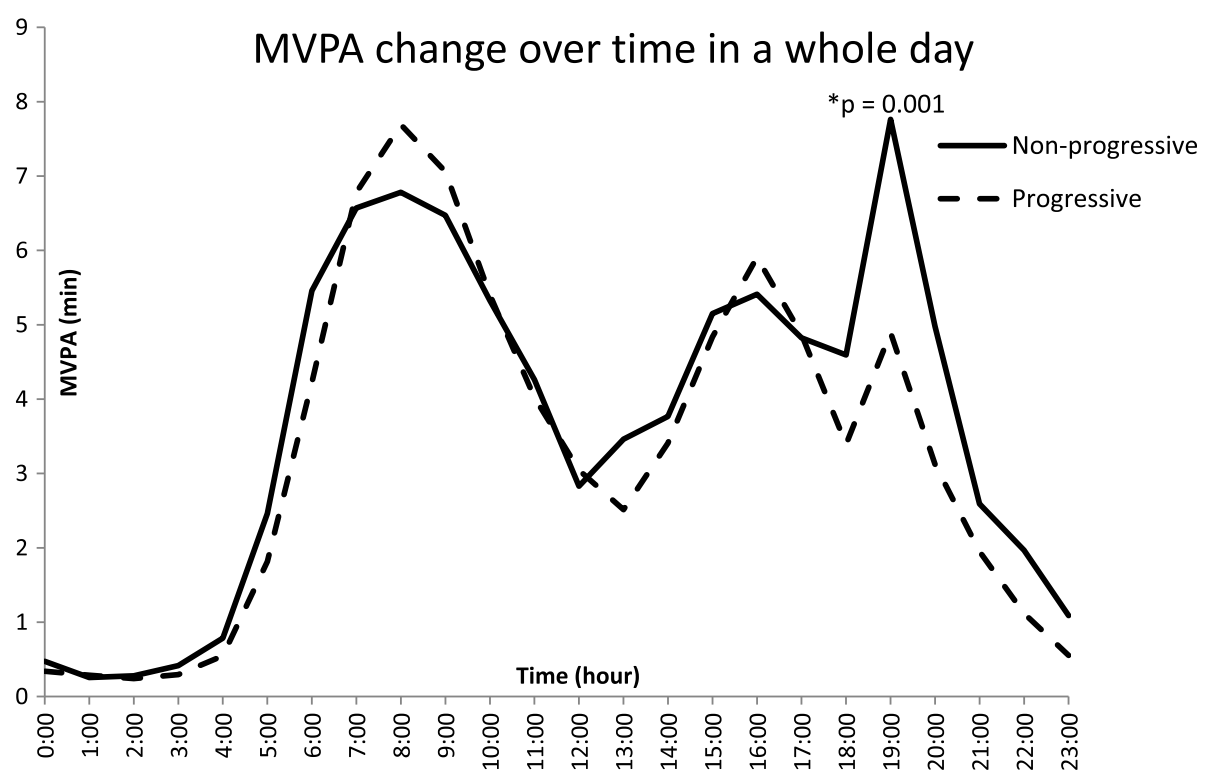

Fig. 1 24-h variation of MVPA time shown (3 wave peaks and 2 wave hollows) for the non-progressive and progressive groups. The MVPA time have significant difference between those two groups during 18:00-20:00 pm, but have no statistical difference during 07:00-09:00 am and 15:00-17:00 pm

\section{Discussion}

This study objectively monitored the 24-h PA of POAG patients to provide a solid portrayal of their daily PA lifestyle. Adjusted OR for MVPA time per 10 min (18: 00-20:00 h) was 0.85 (95\% CI: 0.75-0.97) for subjects with progressive VF defect in comparison to those with non-progressive VF damage, indicating an increase of time spent in MVPA by 10 min decreases the odds of progressive VF defect by $15 \%$. However, daily PA time of different intensity had no statistical association with progressive glaucomatous VF loss. Lee and his team [21] investigated 141 suspects or manifest glaucoma patients and found that increased PA such as walking, MVPA, and non-sedentary activity were associated with slower rates of VF loss. This discrepancy may have arisen from differences in subject characteristics (POAG vs. suspects and manifest glaucoma), measurement of VF progression (progressor vs. the rate of VF loss) and enrollment criteria (without intraocular surgery vs. conditional surgery).

The levels of VF damage may affect patients' daily physical activities. Ramulu [22] monitored 83 glaucoma subjects and 58 controls for their daily minutes of

Table 3 Univariate and multivariable odds ratio and 95\% confidence intervals for VF progression of POAG patients

\begin{tabular}{|c|c|c|c|c|c|c|c|c|}
\hline \multirow[t]{3}{*}{ Parameter } & \multicolumn{4}{|c|}{ Univariate } & \multicolumn{4}{|c|}{ Multivariable } \\
\hline & \multirow[t]{2}{*}{$\mathrm{OR}$} & \multicolumn{2}{|c|}{$95 \% \mathrm{Cl}$} & \multirow[t]{2}{*}{$p$ value } & \multirow[t]{2}{*}{ OR } & \multicolumn{2}{|c|}{$95 \% \mathrm{Cl}$} & \multirow[t]{2}{*}{$p$ value } \\
\hline & & lower & upper & & & lower & upper & \\
\hline MVPA (per 10 mins) (18:00-20:00 pm) & 0.82 & 0.73 & 0.92 & 0.001 & 0.85 & 0.75 & 0.97 & 0.016 \\
\hline $\mathrm{MD}(\mathrm{dB})$ & 1.03 & 1.00 & 1.06 & 0.074 & & & & \\
\hline RNFL $(\mu \mathrm{m})$ & 1.00 & 0.99 & 1.01 & 0.854 & & & & \\
\hline aMAP $(\mathrm{mmHg})$ & 0.96 & 0.94 & 0.98 & $<0.001$ & 0.95 & 0.93 & 0.97 & $<0.001$ \\
\hline $\mathrm{mlOP}(\mathrm{mmHg})$ & 1.02 & 0.96 & 1.08 & 0.511 & & & & \\
\hline Age (yrs) & 1.06 & 1.03 & 1.08 & $<0.001$ & 1.08 & 1.05 & 1.11 & $<0.001$ \\
\hline Gender (male) & 0.67 & 0.48 & 0.96 & 0.027 & 1.15 & 0.75 & 1.75 & 0.520 \\
\hline$C C T(\mu \mathrm{m})$ & 1.00 & 1.00 & 1.01 & 0.719 & & & & \\
\hline SE (D) & 1.14 & 1.07 & 1.22 & $<0.001$ & 1.11 & 1.02 & 1.20 & 0.014 \\
\hline IOP-lowering medications & 1.54 & 1.16 & 2.05 & 0.003 & 2.95 & 2.02 & 4.31 & $<0.001$ \\
\hline
\end{tabular}

$\mathrm{Cl}=$ confidence interval; $M V P A=$ moderate to vigorous physical activity time per ten minutes when patients exercise between 18:00 to 20:00 pm; $M D=$ mean deviation at the baseline; $R N F L=$ average retinal nerve fiber layer detected by OCT at the baseline; $a M A P=$ average mean arterial pressure during all visits, calculated by (systolic blood pressure $+2 \times$ diastolic blood pressure) $\div 3 ; \mathrm{mIOP}=$ mean intraocular pressure during all visits; $S E=$ spherical equivalent; $C C T=$ central corneal thickness; IOP-lowering medications, the number of IOP-lowering medications. Bold values indicate statistical significance with alpha set at 0.05 
MVPA and steps per day, finding that daily PA time was impacted by glaucomatous VF loss. In our study, the chosen eye (worse or progressive eye) VF mean deviations were $-6.22 \pm 6.09 \mathrm{~dB}$ in the non-progressive group and $-7.73 \pm 5.45 \mathrm{~dB}$ in the progressive group $(p=0.084)$ at the time of PA assessment. In addition, the percentage of people with mild to moderate VF defect was 94.40 and $92.60 \%$ in these two groups $(p=0.744)$. Overall, no significant difference in presenting visual acuity was found between the two groups, reducing the possibility that progressors exercise less in the evening due to the severe VF loss.

This is the first study showing a correlation between evening exercise (18:00-20:00 h) and VF loss progression in glaucoma. Circadian rhythms and cyclical physiological changes over a 24-h period may play an important role in the findings as the choice of what time of day to exercise heavily influences physiological variables. Many biological functions have been shown to be timeof-day dependent as their physiological levels fluctuate throughout the day. Ammar A [27] summarized the effects of time-of-day on many physiological functions and how biological parameters adjust in response to increased PA of healthy subjects. In the early evening, individuals have a higher rate of oxidative stress, white blood cell count, homocysteine and muscle damage markers, and a more efficient antioxidant activity than in the early morning. Trabelsi $\mathrm{K}$ [28] explored the diurnal variation $(08: 00 \mathrm{~h}, 14: 00 \mathrm{~h}$ and $18: 00 \mathrm{~h})$ of the same hematological parameters and found that total white blood cell count $(\mathrm{WBC})(p<0.01)$ and neutrophils count $(\mathrm{NE})(\mathrm{p}<0.01)$ have higher resting values in the early evening than in the morning. Previous research has also shown that the impact of repeated sprinting exercises on hematocytes [WBC, NE, lymphocytes (LY) and monocytes $(\mathrm{MO})]$ being dependent on the time-of-day, with greater values obtained in the early evening [29]. Additionally, the effect of time-of-day on many physiological functions can change in response to PA. Studies demonstrate that in the evening, aerobic exercise's time to exhaustion, body temperature, peak oxygen consumption and aerobic system response, are higher in comparison to the same measures in the morning [27, 30, 31]. Evening exercise is better than morning exercise for fat oxidation levels and energy expenditure (EE) as EE and oxygen uptake are higher in the evening $[32,33]$. Nikkhah A [34] also demonstrated that early night exercise can decrease cellular irresponsiveness to insulin, particularly for those overweight or obese. Metabolic levels not only depend on the time-of-day, but also change in response to PA. Therefore, an individual's metabolic levels vary depending on when he or she chooses to exercise. However, the underlying mechanism responsible for the relationship between evening exercise and glaucomatous VF progression remains unclear.
Furthermore, it is widely believed that elevated IOP, vascular dysregulation, and perfusion deficit may lead to glaucoma progression $[35,36]$. Elevated IOP is the only modifiable risk factor for most glaucoma subtypes, including POAG. Decreased ocular perfusion and vascular dysregulation has also been implicated as a major risk factor for glaucoma [37, 38]. Past research has shown that dynamic exercise significantly reduces IOP and increases ocular blood flow. Natsis $K$ [39] found that aerobic exercise can help reduce IOP even when patients are already on various antihypertensive drugs. Hayashi N [40] reported that increasing exercise intensity induces rising retina and choroidal blood flow. Portmann N [15] explored the response of submacular choroid blood flow (ChBF) to isometric exercise and observed that POAG patients have a smaller active regulatory capacity and a larger increase in ChBF after exercising in comparison with their healthy counterparts, indicating that ocular blood flow in POAG patients is more sensitive to exercise. Taken together, exercise not only decreases IOP, but also increases ocular perfusion (ChBF, ocular perfusion pressure and ocular blood pressure), which may explain why more PA is associated with lower risk of glaucomatous progressive VF damage.

Much like other patients with chronic diseases, glaucoma patients are more likely to have depression with a reported prevalence of $10.9 \%$ in comparison with those without glaucoma (6.9\%) [41]. Literature has shown that exercise reduces the incidence of depression or anxiety $[8,42,43]$. For example, Babyak M [44] investigated 156 adult volunteers with major depressive disorder for 10 months, and found that increasing exercise time lowers depression relapse rates in comparison to that of the medication group. Apart from alleviating depression, exercise can also improve sleep quality, reduce morbidity of cardiovascular diseases, boost the immune system, and even promote neuronal plasticity $[8-12,45]$, and thus have comprehensive benefits not only for the eyes, but the entire body.

An advantage of our study includes using a widely recognized accelerometer to.

measure the 24-h PA of patients to provide objective, comprehensive results. Additionally, our study is the first to discuss the relationship of exercise habits and glaucomatous VF progressive defect, finding that more evening exercise was associated with lower odds of VF progression. During the follow-up visits, we collected data on mean IOP and MAP and adjusted them in the multivariable analysis. Furthermore, the diagnosis of POAG was performed clinically, which was more accurate than population-based designs [16, 17]. However, there are several limitations in our work: Firstly, 
our study is an observational study, so we cannot conclude that there is a causal relationship between evening exercise and progressive VF defect. Secondly, the device is not waterproof, and we did not monitor swimming PA time, which may have resulted in a lesser amount of recorded PA time. Anticipating this, we asked all our subjects if they swim regularly and found that $93(94.9 \%)$ never swim, one $(1.0 \%)$ swims regularly in the summertime and four (4.1\%) occasionally swim, indicating this to be a minor flaw. Thirdly, using the monitor to measure the daily PA of the elderly with visual impairment may have some deviations than that in the other elderly. However, to our knowledge, no validity studies exist on tools that measure PA in patients with ocular disorders. Valid and specific tools need to be designed in future studies when measuring daily PA for the elderly with visual impairment. Lastly, age was not matched in the progressive and nonprogressive group and there was no control group without POAG. Although the age factor was adjusted in the multivariate analysis, a health control group could provide a well-balanced comparison between the groups.

\section{Conclusions}

In conclusion, our study found that more evening exercise (MVPA) was associated with lower odds of progressive VF damage in patients with POAG. Our data suggest that exercise habits may play an important role in glaucoma progression, but a prospective, interventional study is needed to confirm our findings.

\section{Abbreviations \\ AL: Axial lengths; BMl: Body-mass index; CCT: Central corneal thickness; ChBF: Choroid blood flow; CNTGS: Collaborative Normal-Tension Glaucoma Study; EE: Energy expenditure; EMGT: Early Manifest Glaucoma Trial; HTG: High-tension glaucoma; IOP: Intraocular pressure; IQR: Interquartile Range; LY: Lymphocytes; MAP: Mean arterial pressure; MO: Monocytes; MVPA: Moderate to vigorous physical activity; NE: Neutrophils count; NTG: Normal tension glaucoma; OR: Odds ratio; PA: Physical activity; POAG: Primary open angle glaucoma; RNFL: Retinal nerve fiber layer; SE: Spherical equivalent; VF: Visual field; WBC: White blood cell; \\ WGPS: Wenzhou Glaucoma Progression Study}

\section{Acknowledgements}

The authors thank Kemi Feng for statistical consultation, Haishuang Hu and Qiangjie Huang for research assistance.

\section{Authors' contributions}

XFP collected and analyzed data, and wrote the manuscript. XW, GFC and $\mathrm{HHC}$ collected data. YBL and JC conceived the study, participated in the discussion and revised the manuscript. KX, AJL, LTH and ZL contributed to discussion and edited the manuscript. All authors have read and approved the final manuscript.

\section{Funding}

This study was supported by Wenzhou Medical University Research Fund No. QTJ13009, Zhejiang Province Health Innovation Talents Project no. 2016025, Key Discipline of the Eye Hospital, School of Ophthalmology and Optometry, Wenzhou Medical University Fund no. ZDZK201808 and the Innovation Research Project of the Eye Hospital of Wenzhou Medical University no. YNCX201308.
Availability of data and materials

The datasets used and/or analyzed during the current study are available from the corresponding author on reasonable request.

Ethics approval and consent to participate

This study was approved by the Institutional Review Board of Wenzhou Medical University (KYK [2018]20).

\section{Consent for publication}

All patients gave informed consent.

\section{Competing interests}

The authors declare that they have no competing interests.

\section{Author details}

'Department of Ophthalmology, Guangdong Provincial Key Laboratory of Malignant Tumor Epigenetics and Gene Regulation, Sun Yat-sen Memorial Hospital, Sun Yat-sen University, Guangzhou, China. ${ }^{2}$ School of

Ophthalmology and Optometry, Eye Hospital, Wenzhou Medical University, No. 270, Xue Yuan Xi Road, Wenzhou 3250027, Zhejiang, China. ${ }^{3}$ Nanjing Sport Institute, No.8 Linggusi Road, Nanjing, Jiangsu, China. ${ }^{4}$ Glaucoma Institute, Wenzhou Medical University, Wenzhou, Zhejiang, China. ${ }^{5}$ Johns Hopkins University School of Medicine, Baltimore, MD 21205, USA.

Received: 25 July 2019 Accepted: 4 February 2020

Published online: 01 March 2020

\section{References}

1. Weinreb RN, Khaw PT. Primary open-angle glaucoma. Lancet. 2004; 363(9422):1711-20.

2. Tham YC, Li X, Wong TY, Quigley HA, Aung T, Cheng CY. Global prevalence of glaucoma and projections of glaucoma burden through 2040: a systematic review and meta-analysis. Ophthalmology. 2014;121(11):2081-90.

3. Heijl A, Bengtsson B, Hyman L, Leske MC, Early Manifest Glaucoma Trial Group. Natural history of open-angle glaucoma. Ophthalmology. 2009; 116(12):2271-6.

4. Anderson DR, Drance SM, Schulzer M. Natural history of normal-tension glaucoma. Ophthalmology. 2001;108(2):247-53.

5. Collaborative Normal-Tension Glaucoma Study Group. Comparison of glaucomatous progression between untreated patients with normal-tension glaucoma and patients with therapeutically reduced intraocular pressures. Am J Ophthalmol. 1998:126(4):487-97.

6. Musch DC, Gillespie BW, Lichter PR, Niziol LM, Janz NK, CIGTS study investigators. Visual field progression in the Collaborative Initial Glaucoma Treatment Study: the impact of treatment and other baseline factors. Ophthalmology. 2009:116(2):200-7.

7. Piercy KL, Troiano RP, Ballard RM, Carlson SA, Fulton JE, Galuska DA, et al. The physical activity guidelines for Americans. JAMA. 2018;320(19):2020-8.

8. Kanamori S, Takamiya T, Inoue S, Kai Y, Tsuji T, Kondo K. Frequency and pattern of exercise and depression after two years in older Japanese adults: the JAGES longitudinal study. Sci Rep. 2018;8(1):11224.

9. Gleeson M. Immune function and exercise. Eur J Sport Sci. 2004;4(3):52-66.

10. Kelley GA, Kelley KS. Exercise and sleep: a systematic review of previous meta-analyses. J Evid Based Med. 2016;10(1):26-36.

11. Leung FP, Yung LM, Laher I, Yao X, Chen ZY, Huang Y. Exercise, vascular wall and cardiovascular diseases: an update (part 1). Sports Med. 2008; 38(12):1009-24.

12. Yung LM, Laher I, Yao X, Chen ZY, Huang Y, Leung FP. Exercise, vascular wall and cardiovascular diseases: an update (part2). Sports Med. 2008;39(1): 45-63.

13. Yan X, Li M, Song Y, Guo J, Zhao Y, Chen W, et al. Influence of exercise on intraocular pressure, Schlemm's canal, and the trabecular meshwork. Invest Ophthalmol Vis Sci. 2016:57(11):4733-9.

14. Qureshi IA. The effects of mild, moderate, and severe exercise on intraocular pressure in glaucoma patients. Jpn J Physiol. 1995;45(4):561-9.

15. Portmann N, Gugleta K, Kochkorov A, Polunina A, Flammer J, Orgul S. Choroidal blood flow response to isometric exercise in glaucoma patients and patients with ocular hypertension. Invest Ophthalmol Vis Sci. 2011; 52(10):7068-73. 
16. Lin SC, Wang SY, Pasquale LR, Singh K, Lin SC. The relation between exercise and glaucoma in a south Korean population-based sample. PLoS One. 2017;12(2):e0171441.

17. Williams PT. Relationship of incident glaucoma versus physical activity and fitness in male runners. Med Sci Sports Exerc. 2009;41(8):1566-72.

18. Collings PJ, Brage S, Ridgway CL, Harvey NC, Godfrey KM, Inskip HM, et al. Physical activity intensity, sedentary time, and body composition in preschoolers. Am J Clin Nutr. 2013;97(5):1020-8.

19. Bouten CV, Koekkoek KT, Verduin M, Kodde R, Janssen JD. A triaxial accelerometer and portable data processing unit for the assessment of daily physical activity. IEEE Trans Biomed Eng. 1997;44(3):136-47.

20. Cheung VH, Gray L, Karunanithi M. Review of accelerometry for determining daily activity among elderly patients. Arch Phys Med Rehabil. 2011;92(6): 998-1014.

21. Lee MJ, Wang J, Friedman DS, Boland MV, De Moraes CG, Ramulu PY. Greater physical activity is associated with slower visual field loss in glaucoma. Ophthalmology. 2019;126(7):958-64.

22. Ramulu PY, Maul E, Hochberg C, Chan ES, Ferrucci L, Friedman DS. Realworld assessment of physical activity in glaucoma using an accelerometer. Ophthalmology. 2012;119(6):1159-66.

23. ActiGraph Monitor [EB/OL]. http://www.theactigraph.com/article/researchdatabase.

24. Sasaki JE, John D, Freedson PS. Validation and comparison of ActiGraph activity monitors. J Sci Med Sport. 2011;14(5):411-6.

25. Bengtsson B, Lindgren A, Heijl A, Lindgren G, Asman P, Patella M. Perimetric probability maps to separate change caused by glaucoma from that caused by cataract. Acta Ophthalmol Scand. 1997;75(2):184-8.

26. Katz J. A comparison of the pattern- and total deviation-based Glaucoma Change Probability programs. Invest Ophthalmol Vis Sci. 2000;41(5):1012-6.

27. Ammar A, Chtourou H, Souissi N. Effect of time-of-day on biochemical markers in response to physical exercise. J Strength Cond Res. 2017;31(1): 272-82.

28. Ammar A, Chtourou H, Trabelsi K, Padulo J, Turki M, El Abed K. Temporal specificity of training: intra-day effects on biochemical responses and Olympic-Weightlifting performances. J Sports Sci. 2015;33(4):358-68.

29. Hammouda O, Chtourou H, Chahed H, Ferchichi S, Kallel C, Miled A, et al. Diurnal variations of plasma homocysteine, total antioxidant status, and biological markers of muscle injury during repeated sprint: effect on performance and muscle fatigue--a pilot study. Chronobiol Int. 2011;28(10): 958-67.

30. Yamanaka Y, Honma Kl, Hashimoto S, Takasu N, Miyazaki T, Honama S. Effects of physical exercise on human circadian rhythms. Sleep Biol Rhythms. 2006;4(3):199-206.

31. Hill DW, Borden DO, Darnaby KM, Hendricks DN, Hill CM. Effect of time of day on aerobic and anaerobic responses to high-intensity exercise. Can J Sport Sci. 1992;17(4):316-9.

32. Azizi M, Rahmani-Nia F, Mohebbi H. Cortisol responses and energy expenditure at different times of day in obese Vs. lean men. World J Sport Sci. 2012;6(3):314-20.

33. Hamid M, Mohammad A. Maximal fat oxidation at the different exercise intensity in obese and normal weight men in the morning and evening. J Human Sport Exer. 2011;6(1):49-58.

34. Nikkhah A. Evening exercise to prevent central adiposity and cardiometabolic diseases. Int J Diabetol Vasc Dis Res. 2015;3(6):113-4.

35. Heijl A, Leske MC, Bengtsson B, Hyman L, Bengtsson B, Hussein M. Reduction of intraocular pressure and glaucoma progression: results from the Early Manifest Glaucoma Trial. Arch Ophthalmol. 2002;120(10):1268-79.

36. Liu CH, Su WW, Shie SS, Cheng ST, Su CW, Ho WJ. Association between peripheral vascular endothelial function and progression of open-angle glaucoma. Medicine (Baltimore). 2016;95(10):e3055.

37. Risner D, Ehrlich R, Kheradiya NS, Siesky B, McCranor L, Harris A. Effects of exercise on intraocular pressure and ocular blood flow: a review. J Glaucoma. 2009;18(6):429-36

38. Caprioli J, Coleman AL, Blood flow in Glaucoma discussion. Blood pressure, perfusion pressure, and glaucoma. Am J Ophthalmol. 2010;149(5):704-12.

39. Natsis K, Asouhidou I, Nousios G, Chatzibalis T, Vlasis K, Karabatakis V. Aerobic exercise and intraocular pressure in normotensive and glaucoma patients. BMC Ophthalmol. 2009;9:6.

40. Hayashi N, Ikemura T, Someya N. Effects of dynamic exercise and its intensity on ocular blood flow in humans. Eur J Appl Physiol. 2011;111(10): 2601-6.
41. Wang SY, Singh K, Lina SC. Prevalence and predictors of depression among participants with glaucoma in a nationally representative population sample. Am J Ophthalmol. 2012;154(3):436-44.e2.

42. Ströhle A. Physical activity, exercise, depression and anxiety disorders. J Neural Transm (Vienna). 2009;116(6):777-84.

43. Lawlor DA, Hopker SW. The effectiveness of exercise as an intervention in the management of depression: systematic review and meta-regression analysis of randomised controlled trials. BMJ. 2001;322(7289):763-7.

44. Babyak M, Blumenthal JA, Herman S, Khatri P, Doraiswamy M, Moore K, et al. Exercise treatment for major depression: maintenance of therapeutic benefit at 10 months. Psychosom Med. 2000;62(5):633-8.

45. Sandrow-Feinberg HR, Houlé JD. Exercise after spinal cord injury as an agent for neuroprotection, regeneration and rehabilitation. Brain Res. 2015; 1619:12-21
Ready to submit your research? Choose BMC and benefit from:

- fast, convenient online submission

- thorough peer review by experienced researchers in your field

- rapid publication on acceptance

- support for research data, including large and complex data types

- gold Open Access which fosters wider collaboration and increased citations

- maximum visibility for your research: over $100 \mathrm{M}$ website views per year

At BMC, research is always in progress.

Learn more biomedcentral.com/submissions 